\title{
HIV-1 Genotypes Related to Failure of Nelfinavir as the First Protease Inhibitor Treatment
}

Unaí Tupinambás ${ }^{1}$, Agdemir Aleixo ${ }^{3,4}$ and Dirceu Greco ${ }^{2,3}$

\begin{abstract}
${ }^{1}$ Belo Horizonte Secretary of Health; ${ }^{2}$ Pos-Graduation Course in Tropical Medicine and Immunology and Molecular Biology Laboratory, School of Medicine, Federal University of Minas Gerais; ${ }^{3}$ State Secretary of Health of Minas Gerais; Belo Horizonte, MG, Brazil
\end{abstract}

\begin{abstract}
Combined antiretroviral therapy results in sustained viral suppression and a decrease in mortality and morbidity due to HIV infection. Intrinsic strength, durability and absence of crossresistance are key factors in the selection of antiretrovirals. Failure with nelfinavir has been associated with two protease gene mutations, D30N and L90M. The D30N mutation does not result in cross-resistance with other protease inhibitors, and it decreases viral fitness. In order to check for this mutation after failure with nelfinavir, the $246 \mathrm{HIV}-1$ genotyping test was performed on virus samples from 55 patients with failure of nelfinavir as the first protease inhibitor. Most $(84 \%)$ of the viral strains were of subtype B. Nucleosides associated with mutations (NAM) were observed in $80 \%$ of the tests; no INS69, complex 151, K65R and L74V mutations, which give multi-resistance to nucleoside analogue reverse transcriptase inhibitors to tenofovir and DDI, respectively, were observed. In the tests for protease gene mutations, the D30N mutation was found in $57 \%$, $\mathrm{L90M}$ in $18 \%$ and the wild-type virus in $25 \%$. These data are similar to published reports, showing that alternative therapies used after failure with nelfinavir may be more successful, as the D30N mutation does not cause cross-resistance to other protease inhibitors. Key Words: HIV-1, protease inhibitors, nelfinavir.
\end{abstract}

Combined antiretroviral therapy results in significant viral suppression [1,2], with a decrease in morbidity and mortality related to HIV-1 infection [3,4]. However, therapeutic efficacy has been threatened by the appearance of resistant viral strains [5,6], which occur more frequently in patients receiving a decreased efficacy antiretroviral regimen (e.g., double therapy) or in those with unsatisfactory conformance $[7,8]$. While intrinsic strength and the duration of the efficacy of the treatment regime are the main factors taken into consideration for the selection of antiretrovirals, the absence of cross-resistance, in order to preserve future

Received on 18 May 2005; revised 28 July 2005.

Address for correspondence: Dr. Unaí Tupinambás. Rua Dona Cecília 81/301. Serra Belo Horizonte. Zip Code 30220-070. Phone: 55 31-3248-9822; fax: 55 31-32817418. E-mail: unai@uai.com.br

The Brazilian Journal of Infectious Diseases 2005;9(3):324-329 (C) 2005 by The Brazilian Journal of Infectious Diseases and Contexto Publishing. All rights reserved. treatment options, is another important aspect of treatment choice [9].

Different mutations may be selected for after exposition to different antiretrovirals within the same class. Among the mutations in the protease gene, the primary ones appear in the initial phase of therapeutic failure. Sometimes, they are specific to each drug; while the secondary (or compensatory) mutations appear later and have similar effects on the drugs of this class $[10,11]$. When there is an accumulation of these mutations, the possibility of cross-resistance is increased.

The replacement of asparagine by aspartic acid at position 30 of the protease gene (D30N) is the main mutation that appears after failure with nelfinavir; and according to a number of authors [9,12-14], it is the only one caused by this drug, thus not inducing crossresistance. Also, the D30N mutation decreases the capacity of viral replication (viral fitness), when compared to wild-type viral strains [15]. However, the L90M mutation (replacement of methionine by leukine), 
which occurs in some cases of therapeutic failure with nelfinavir [9,12-14], causes cross-resistance to all drugs of this class [16,17]; the therapeutic response in these cases is worse than in patients presenting the D30N mutation [18]. In this situation, a change to a regimen with non-nucleoside analogue reverse transcriptase inhibitors, or with protease inhibitors boosted by ritonavir, seems to be the best option.

With the establishment of the National Net of HIV Genotyping (RENAGENO) by the National Program of STD/AIDS of the Ministry of Health, the profile of the mutations occurring after failure of nelfinavir as the first protease inhibitor in our setting became available. This net has been operating since February 2002. Within this context, the Project "GERAIS" - Grupo de Estudos em Resistência aos anti-retrovirais [Study Group on Resistance to Antiretrovirals] was created, with the main purpose of contributing to the evaluation of the efficacy of this new technology. Our study was proposed in this context, with the primary objective of evaluating the characteristics of the HIV-1 genotyping test after therapeutic failure. Its secondary objectives include: phylogenetic analysis of HIV-1 and determination the of protease gene mutations, detection of mutations related to nucleosides (NAM) and the time of use of ARV, and the number of secondary mutations and their relation to duration of treatment.

\section{Materials and Methods}

Two hundred and forty six HIV-1 genotyping tests were made in Molecular Biology and Immunology Laboratory of the Department of Infectious and Parasitic Diseases of the School of Medicine of the Federal University of Minas Gerais from February 2002 to March 2004. Only the tests of patients at first failure, having nelfinavir as the first protease inhibitor (PI), preceded by double or triple therapy with nonnucleoside analogue reverse transcriptase inhibitors (nNRTI) $(\mathrm{n}=55)$ were included in our study.

The mutations were interpreted using the manufacturer's data (Applied Biosystems, Foster City, California, USA), the International AIDS Society analysis [19], and the RENAGENO algorithm. The mutations at the following positions were considered primary mutations in the protease gene: $30,46,48$, 50, 82 (except for V82I), 84 and 90, and the ones at positions 10, $2024,32,33,36,47,53,54,71,73$, 77 and 88 were considered secondary mutations. In the case of the nucleoside analogue reverse transcriptase inhibitors (NRTI), mutations in the transcriptase gene in the following positions were considered: 41, 44, 62, $65,67,69,70,74,75,77,115,116,118,151,184$, 210,215 and 219 . The mutations in the following positions were considered to involve non-nucleoside analogue reverse transcriptase inhibitors (nNRTI): 100, 103, 108, 181, 190, 225 and 236.

The descriptive measures were calculated as percentages, with mean, minimum, maximum and standard deviation.

The comparisons of the time (duration) of antiretroviral therapy (TARV) were performed using the Mann-Whitney test or the Kruskal-Wallis test. The alpha level for significance was $5 \%$.

\section{Results}

Fifty-five HIV-1 patients for which nelfinavir failed as the first PI were genotyped The TARV ranged between 10 and 72 months, with a mean of 34 months, a median of 30 months and standard deviation of 17.6.

Among the 55 patients, 55\% (30) had already used double therapy with NRTI or triple therapy with nNRTI and the rest $(46 \%$ - 25) were in first therapeutic failure. In the evaluation of HIV subtype (Figure 1), most of them ( $84 \%$ ) had subtype B, $7 \%$ had subtype F and $9 \%$ had a recombinant subtype.

Among the patients, $82 \%$ (45) presented reverse transcriptase (RT) gene mutations. The mutations associated with nucleosides (NAM) were found in 35 patients; most of them had three to five NAM. The mutation at position 184 was found in 38 patients (69\%). The mutations at positions 69 (insertion), 151, 65 and 74, which are related to multi-resistance to NRTI (INS69 and complex 151), resistance to tenofovir and DDI, respectively, were not found (Table 1). 
Figure 1. Characterization of 43 patients according to HIV subtype.

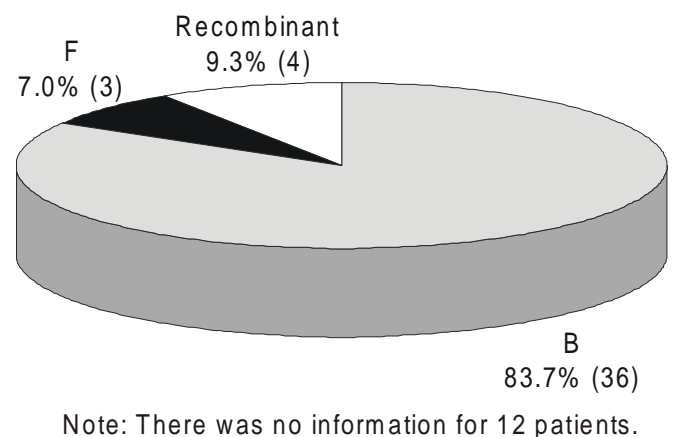

Figure 2. Profile of the primary mutations after failure with nelfinavir

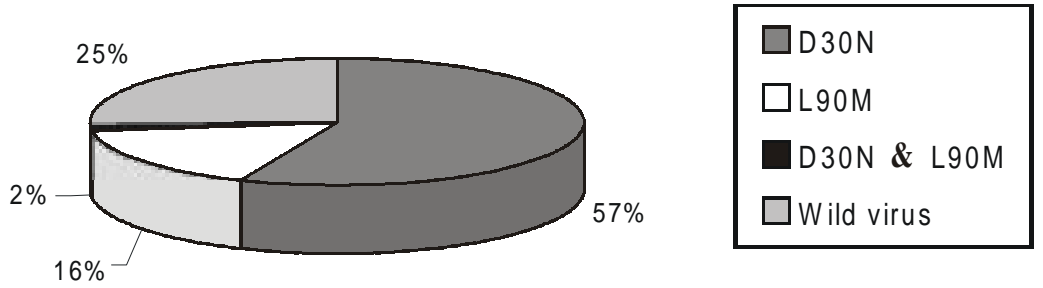

Table 1. Characterization of mutations in the reverse transcriptase gene $(\mathrm{N}=55)$

\begin{tabular}{lrr}
\hline & \multicolumn{2}{c}{ Frequency } \\
\hline Variables & N & $\%$ \\
$\quad$ Wild virus & 10 & 18.2 \\
Presence of NAM & 35 & 79.5 \\
$\quad$ No information & 1 & \\
Number of NAM & & \\
1 to 2 & 11 & 31.4 \\
3 to 5 & 22 & 62.9 \\
Above 5 & 2 & 5.7 \\
Presence of M184V & 38 & 69.1 \\
\hline
\end{tabular}

$\mathrm{NAM}=$ nucleoside associated mutations. 
Table 2. Characterization of protease gene mutations

\begin{tabular}{llr}
\hline & \multicolumn{2}{c}{ Frequency } \\
\cline { 2 - 3 } Variables & $\mathbf{N}$ & \% \\
\hline Presence of primary mutation to nelfinavir & 41 & 74.5 \\
$\quad$ D30N & 31 & 75.6 \\
L90M & 9 & 22.0 \\
$\quad$ Both & 1 & 2.4 \\
Presence of secondary mutations & & \\
$\quad$ Yes & 48 & 100.0 \\
Number of secondary mutations & & \\
$\quad$ Up to 2 & 13 & 27.1 \\
$\quad$ Above 2 to 4 & 25 & 52.1 \\
5 or more & 10 & 20.8 \\
$\quad$ Total & 48 & 100.0 \\
\hline
\end{tabular}

Table 3. Characterization of the patients according to the primary mutation to nelfinavir and the HIV subtype

\begin{tabular}{llrlrlrc}
\hline & \multicolumn{6}{c}{ Primary mutation to nelfinavir } \\
\cline { 2 - 8 } Subtype & \multicolumn{2}{c}{ D30N } & \multicolumn{2}{c}{ L90M } & \multicolumn{2}{c}{ Both } & \\
\cline { 2 - 8 } & $\mathbf{N}$ & \% & N & \% & N & \% & Total \\
B & 23 & 85.2 & 4 & 14.8 & 0 & 0.0 & 27 \\
F & 1 & 100.0 & 0 & 0.0 & 0 & 0.0 & 1 \\
Recombinant & 1 & 33.3 & 1 & 33.3 & 1 & 33.3 & 3 \\
Total & 25 & 5 & 1 & 31 & & & \\
\hline
\end{tabular}

Note: 10 cases with no information.

Among the 55 patients, $41(75 \%)$ presented primary mutations related to nelfinavir; most of these had the D30N mutation $(31=57 \%)$ (Figure 2, Table $2)$. No significant relation was found between the characteristic of the primary mutation to nelfinavir and the HIV-1 subgroup (Table 3). The mean TARV tended to increase as the number of secondary mutations to the PI increased (Table 4).

\section{Discussion}

Most patients had HIV-1 subtype b, though a phylogenetic analysis was not been performed in 12 patients. In the analysis of reverse transcriptase gene mutations, the mutations related to phenotypic multiresistance to the drugs of this class, INS69 and complex Q151M, were not seen. The K65R and L74V mutations, related to phenotypic resistance to tenofovir, were also not seen.

Among the primary mutations related to nelfinavir, D30N was observed in 31 (57\%) of the 55 patients. No protease gene mutation was seen in the remaining 14 patients. Various other studies $[9,12,13,14]$ have also shown that this mutation is the most frequent in cases of failure of nelfinavir therapy; there was low cross-resistance with other PIs and facilitated therapeutic rescue. However, though there is indication of a decreased viral replication capacity with the D30N mutation [15], the clinical benefits of this condition are still unknown. 
Table 4. Characterization of patients according to the time of use of antiretroviral drugs, considering the number of secondary mutations to the protease inhibitor (PI)

\begin{tabular}{lcccccc}
\hline $\begin{array}{l}\text { Mutations } \\
\text { for PI }\end{array}$ & N & Minimum & Maximum & Median & Mean & SD \\
\cline { 2 - 7 } & 13 & 13 & 52 & 20 & 23.9 & 11.1 \\
Up to 2 & 25 & 10 & 69 & 33 & 36.0 & 18.3 \\
From 2 to 4 & 10 & 14 & 72 & 34 & 41.1 & 21.5 \\
5 or more & & & & & &
\end{tabular}

Note: $\mathrm{p}=0.076$, Kruskal-Wallis test.

There was a tendency towards a greater number of secondary mutations with increased TARV, though it was not statistically significant.

In conclusion, it remains unclear whether there should be an immediate ARV substitution after the finding of this mutation, because of the risk of quickly exhausting the still-limited available therapeutic arsenal. On the other hand, such a substitution could avoid an accumulation of secondary mutations, avoiding resistance to all drugs of this class. It will be necessary to pursue clinical end points to answer these questions.

\section{Acknowledgements}

National Program of STD/AIDS - MS/Brazil and Department of Genetic of the Federal University of Rio de Janeiro, Rio de Janeiro, RJ, Brazil.

\section{References}

1. Perelson A.S., Essunger P., Cao Y., et al. Decay characteristics of HIV-1 infected compartments during combination therapy. Nature 1997;387:18891.

2. Gulick R.M., Mellors J.W., Havlir D., et al. Treatment with indinavir, zidovudine, and lamivudine in adults with human immunodeficiency virus infection and prior antiretroviral therapy. N Eng J Med 1997; 337:734-9.

3. Marins J.R., Jamal L.F., Chen S.Y., et al. Dramatic improvement in survival among adult Brazilian Aids patients. AIDS 2003; 17:1675-82.
4. Palella F.J., Kathellen M.D., Anne C.M., et al. Decline Morbidity among patients with Advanced Human Immunodeficiency Virus Infection. N Engl L Med 1998;338:853-60.

5. Shafer R.W., Winters M.A., Palmer S., et al. Multiple concurrent reverse transcriptase and protease mutations and multidrug resistance of HIV-1 isolates from heavily treated patients. Ann Intern Med 1998;128:906-11.

6. Eastman P.S., Mittler J., Kelso R., et al. Genotypic changes in human immunodeficiency virus type 1 associated with loss of suppression of plasma viral RNA levels subjects treated with ritonavir (Norvir) monotherapy. J Virol 1998;72:5154-64.

7. Haubrich R.H., Little S.J., Currier J.S., et al. The value of patient-reported adherence to antiretroviral therapy in predicting virologic and immunologic response. AIDS 1999; 13:1099-107.

8. Deeks S., Hecht F., Swanson M., et al. HIV RNA and CD4 cell count response to protease inhibitor therapy in an urban AIDS Clinic: Response to both initial and salvage therapy. AIDS 1999;13:F35-F43.

9. Kemper C.A., Witt M.D., Keiser P.H., et al. Sequencing of protease inhibitor therapy: insights from an analysis of HIV phenotypic resistance in patients failing protease inhibitors. AIDS 2001; 15:609-15.

10. Rodrigues-Rosado R., Briones C., Soriano V. Introduction of drug resistance testing in clinical practice. AIDS 1999; 13:1007-14.

11. Harrigan R., Coté H. Clinical utility of testing HIV for drug resistance. Clin Infect Dis 2000;30(Suppl. 2):117-22.

12. Patick A.K., Duran M., Cao Y., et al. Genotypic and phenotypic characterization of human immunodeficiency virus type 1 variants from patients treated with the protease inhibitor nelfinavir. Antimicrob Agents Chemother 1998;42(10):2637-44.

13. Clotet B., Ruiz L., Matinez-Picado J., et al. Prevalence of HIV Protease Mutations on Failure of NelfinavirContaining HARRT: A Retrospective Analysis of Four Clinical Studies and Two Observational Cohorts. HIV Clin Trials 2002;3:316-23. 
14. Tebas P., Patick A., Kane E., et al. Virologic response to a ritonavir-saquinavir-containing regimen in patient who had previously failed nelfinavir. AIDS 1999;13:F23-F8.

15. Martinez-Picado J., Savana A.V., Sutton L., et al. Replicative fitness of protease inhibitor-resistance mutants of human immunodeficiency virus type 1 . J Virol 1999;73:3744-52.

16. Condra J., Holder D., Schleif W., et al. Genetic correlates of virological response to an indinavir-containing salvage regimen in patients with nelfinavir failure. Antiviral Ther 1999;4 (Suppl. 1): 44.

17. Atkinson B., Isaacson J., Knowles M., et al. Correlation between HIV genotypic resistance and virologic response in patient receiving nelfinavir monotherapy or nelfinavir with lamivudine and zidovudine. J Infect Dis 2000; $182: 420-7$.

18. Saah A., Haas D.W., Rhodes R., et al. Virologic response to indinavir (IDV), efavirenz (EFV) and adefovir (ADV) among patients failing nelfinavir (NFV). In: Program and abstracts of the WIII International Conference on AIDS; July 9-14, 2000; Durban, South Africa. Abstract WePeB4169.

19. International AIDS society-USA resistance Mutations Project Panel. Drug resistance mutations in HIV-1. http:/ /wwwiasusa.org. Accessed October 30, 2003. 